${ }^{1}$ Escuela de Enfermería Pontificia Universidad Católica de Chile. Santiago, Chile. ${ }^{2}$ Sociedad Chilena de Enfermería en Diálisis y Trasplante. aEnfermera. ${ }^{\mathrm{b}}$ Enfermera matrona.

Proyecto financiado por la Dirección de Investigación de la Escuela de Enfermería, Pontificia Universidad Católica de Chile y Sociedad Chilena de Enfermera en Diálisis y Trasplante Renal (SENFERDIALT).

Las autoras no declaran conflicto de intereses.

Recibido el 23 de febrero de 2017, aceptado el 3 de agosto de 2017.

Correspondencia a: María Cecilia Arechabala Avda. Vicuña Mackenna 4860, Macul, Santiago de Chile. Fono: 56-23545831 marechab@uc.cl

\section{Carga laboral de las enfermeras en Unidades de Hemodiálisis Crónica según dependencia y riesgo de los pacientes}

\author{
SILVIA BARRIOS ${ }^{1, \mathrm{a}}$, MARÍA ISABEL CATONI ${ }^{1, \mathrm{~b}}$, \\ MARÍA CECILIA ARECHABALA ${ }^{1, a}$, EUGENIA PALMA ${ }^{1, a}$, \\ YOLANDA IBACACHE ${ }^{2, a}$, JOANNA RICHARD ${ }^{2, a}$
}

\section{Nurses' workload in hemodialysis units}

Background: Knowing nurses' workload in hemodialysis units allows a better organization of human resources. Aim: To determine nurses' workload according to dependence and risk profiles of patients undergoing chronic hemodialysis. Material and Methods: One hundred fifty one patients from five hemodialysis units were selected and categorized in terms of dependence and risk using the instrument "Care according to dependence and risk in hemodialysis (CUDYR-DIAL). Nurses' activities for direct and indirect care and the time taken to complete those activities were measured. Results: Per patient, nurses used $36.5 \pm 10.7$ min for direct and 23.6 min for indirect care. The time required for direct care of patients at high risk with partial dependence and at high risk and partial self-sufficiency was 41.2 and 40.3 minutes respectively. Conclusions: During a dialysis session, nurses occupy $60 \%$ of their time providing direct care, mainly directed to patients categorized as being of high risk-partial dependence and high risk-partial self-sufficiency.

(Rev Med Chile 2017; 145: 888-895)

Key words: Dependency; Nurses; Renal Dialysis; Risk; Workload.
C hile al 2016 tenía 19.904 pacientes en Hemodiálisis Crónica (HDC), siendo el 90\% beneficiario del sistema de Garantías Explícitas en Salud (GES). Esta terapia consume cerca del 22\% del presupuesto GES, aproximadamente 170 mil millones de pesos anuales ${ }^{1-3}$.

El cuidado de los pacientes sometidos a HDC requiere de un equipo multidisciplinario, nefrólogo, enfermera, nutricionista, entre otros ${ }^{4}$. En particular se requiere que la enfermera tenga habilidades interpersonales, competencias técnicas y clínicas para otorgar una atención segura y de calidad $^{5}$.

El personal de enfermería representa cerca del $45 \%$ de la fuerza de trabajo de una Unidad de Hemodiálisis (UHD), siendo parte de los costos directos de la diálisis ${ }^{6,7}$. Determinar la dotación de enfermeras para garantizar la seguridad del paciente sigue siendo una decisión importante que deben tomar los administradores de salud ${ }^{8}$.

En Chile, las UHD se rigen por el "Reglamento sobre Centros de Diálisis", promulgado por el Ministerio de Salud en 1994, el cual establece un máximo de seis pacientes por enfermera en las UHD $^{9}$. Han trascurrido 23 años desde su promulgación encontrando ahora una mayor proporción de pacientes mayores de 60 años, con más comorbilidades, que permanecen por más años en hemodiálisis, muchos de los cuales utilizan catéter venoso permanente y presentan alguna limitación de su actividad ${ }^{1,10}$.

Respecto a la dotación de enfermeras la litera- 
tura muestra que una alta proporción de pacientes por enfermera en UHD se asocia a un aumento de tareas necesarias que se dejan de realizar $y$, a un aumento en la probabilidad de que ocurran eventos adversos como hipotensión, acortamiento de los tratamientos y reclamos de los pacientes ${ }^{11,12}$. Por otro lado, debido al envejecimiento, aumento de la comorbilidad y discapacidad física, han aumentado los pacientes dependientes que requieren un mayor número de intervenciones de enfermería para garantizar la calidad de atención ${ }^{13,14}$.

La relación paciente/enfermera en UHD es variada en América Latina y el mundo. En Argentina la Ley 22.853 del año 1977, que norma la organización y funcionamiento de la práctica dialítica dicta que la dotación de personal no debe ser menor a cinco pacientes por técnico en diálisis, sin hacer diferencias entre enfermeras, auxiliares y técnicos ${ }^{15}$. Por otra parte, el convenio colectivo del año 1975, que reúne a personas que trabajan en diferentes instituciones de salud establece una enfermera/o por cada dos pacientes ${ }^{16}$.

En Brasil, la Ley Orgánica 15/1999 de 2014, recomienda una enfermera por cada 35 pacientes en HD y un técnico por cada cuatro pacientes ${ }^{17}$. Sin embargo, el Consejo Federal de Enfermería (COFEN) de 2017, establece que 33\% deben ser enfermeros y $67 \%$ técnicos como proporción mínima de profesional/paciente/turno ${ }^{18}$.

Asimismo, en Europa la norma de la Comunidad Autónoma de Cataluña-España establece entre los requisitos técnicos para la autorización sanitaria de los centros y UHD, un mínimo de una enfermera por cada cuatro o cinco pacientes por turno $^{19}$. Por otra parte, la Comunidad Autónoma de Madrid establece una enfermera por cada cuatro puestos de trabajo ${ }^{20}$.

Francia exige la presencia permanente durante la hemodiálisis (HD) de al menos una enfermera por cada cuatro pacientes y una auxiliar por cada ocho $^{21}$. Por último, un estudio que compara la práctica de cuidado renal en Europa, donde participaron ocho países (276 centros, con 21.861 pacientes) mostró que la razón paciente/enfermera (incluyendo enfermeras y asistentes) variaba en promedio entre 2,3 (Noruega) y 4,2 (Bélgica) ${ }^{22}$.

Hay diferentes herramientas de medición de carga de trabajo que han sido utilizadas en enfermería. En general pueden agruparse en dos métodos: basados en la actividad o en la dependencia. Los primeros miden las actividades y determinan el tiempo que se ocupa en realizarlas, por lo que están centrados en el cuidado que se otorga ${ }^{8}$. Los segundos clasifican a los pacientes en base a indicadores clínicos críticos, estableciendo categorías que reflejan el nivel de demanda de ellos, centrándose por lo tanto en las necesidades de los pacientes $^{8}$. Ambos no consideran las actividades de enfermería denominadas indirectas. Así, para evaluar la carga de trabajo de enfermería se debe considerar: dependencia del paciente y actividades de enfermería directas e indirectas ${ }^{8}$.

Estudios realizados en Chile han determinado la dotación de personal de enfermería en unidades médico quirúrgicas a través de dos variables, las actividades que realiza el personal de enfermería y los perfiles de los pacientes según su grado de dependencia y riesgo $\mathrm{O}^{23-25}$.

Así, el presente proyecto pretende determinar la carga laboral de la enfermera según perfil de dependencia y riesgo de los pacientes en UHD de Chile.

\section{Pacientes y Método}

Estudio de corte transversal realizado en la Región Metropolitana (RM). Se seleccionaron en forma intencionada cinco UHD, considerando la zona geográfica (oriente, poniente, sur, norte, centro) y el turno; dado que la primera se relaciona con el estrato socioeconómico y la segunda con el grado de dependencia de los pacientes.

El universo estuvo constituido por 595 pacientes, seleccionándose aleatoriamente, con 95\% de confianza y $80 \%$ de potencia una muestra de 151 pacientes, estratificados por UHD y turno, (Figura 1). A los que aceptaron participar se les aplicó el mini-mental de Folstein, y en los casos que obtenían un puntaje inferior a 13 puntos se solicitó autorización a su representante, frente a la negativa de participar fueron reemplazados por otro paciente. Todos los participantes firmaron un consentimiento informado.

Este estudio fue aprobado por el Comité Ético-Científico del Servicio de Salud Metropolitano Sur Oriente y por el Comité de Ética de la Facultad de Medicina de la Pontificia Universidad Católica de Chile.

Las variables del estudio fueron: características de los pacientes; grado de dependencia y riesgo; y carga laboral de la enfermera. 


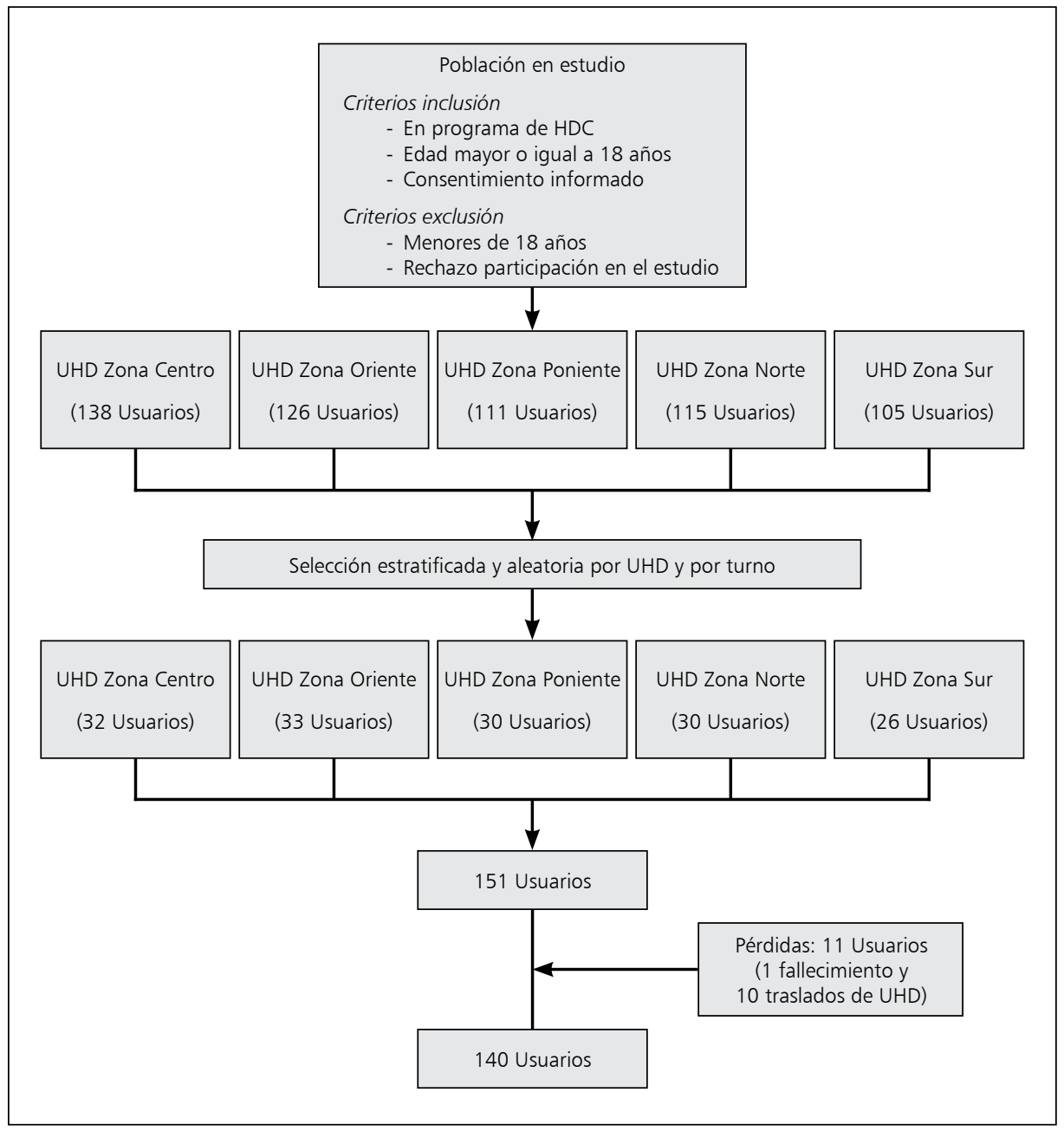

Figura 1. Diagrama de selección de los participantes.

Las características de los participantes se recolectaron de las fichas clínicas: edad, sexo, nivel educacional, causa Insuficiencia Renal Crónica Terminal (IRCT), estado nutricional, acceso vascular, terapia medicamentosa y antecedentes de discapacidad física.

El grado de dependencia y riesgo se midió a través del CUDYR-DIAL adaptado y validado en $\mathrm{HDC}^{14}$. Este mide dos dimensiones: dependencia y riesgo. La dependencia indica el grado de disfuncionalidad o discapacidad del paciente para realizar las actividades de autocuidado, y el riesgo la eventualidad de que el paciente pueda sufrir algún evento adverso o complicación ${ }^{23,24}$. Los cuidados que identifican dependencia son: a) confort y bienestar; b) movilización y trans- porte; c) alimentación; d) eliminación; e) apoyo psicológico y emocional y f) vigilancia. Los cuidados que identifican riesgo son: a) signos vitales; b) balance hídrico; c) oxígenoterapia; d) acceso vascular, e) intervenciones profesionales; f) piel y curaciones; g) administración de fármacos y h) elementos invasivos.

Cada cuidado a su vez tiene tres a cinco ítemes que describen la atención específica que puede requerir el paciente, con un puntaje que va de $0 \mathrm{a}$ 3 puntos. Donde 0 representa el mínimo cuidado, menor dependencia y bajo riesgo; y 3 representa el máximo cuidado, es decir, mayor dependencia y máximo riesgo ${ }^{14}$.

Así, en dependencia el puntaje mínimo es 0 y máximo 18 puntos. En riesgo el puntaje mínimo 
es 0 y máximo 24 puntos. A mayor puntaje mayor dependencia y/o mayor riesgo ${ }^{14}$.

Finalmente, combinando los puntajes obtenidos en las dimensiones de dependencia y riesgo se establecen 12 perfiles de pacientes, desde "máximo riesgo-dependencia total" hasta "bajo riesgo-autosuficiencia parcial" 14 .

El instrumento CUDYR-DIAL fue aplicado por enfermeras de las UHD capacitadas en el mismo. La aplicación se realizó en tres oportunidades a cada participante, ya que el estado de salud y necesidades pueden variar entre una diálisis y otra. Para la categorización de cada paciente, se promediaron los valores obtenidos en cada aplicación.

La carga laboral consideró las intervenciones que realiza la enfermera y el tiempo que ocupa en ellas, distinguiendo cuidados directos e indirectos. Los primeros consideran actividades que requieren contacto con el paciente y/o familia, y los segundos actividades que se realizan para todos los pacientes y que no requieren contacto con ellos.

Para clasificar los cuidados en directos e indirectos se realizaron dos grupos focales con enfermeras de las UHD que participaron en el estudio, obteniéndose un listado que fue validado por enfermeras expertas que no participaban en el estudio.

En las actividades clasificadas como cuidados directos se incluyó: apoyo psicosocial y emocional, higiene y confort, manejo de acceso vascular, seguridad del paciente, cuidados de vigilancia, administración de medicamentos, cuidado de soporte crítico, intervenciones profesionales derivadas de la terapia, medición de parámetros clínicos y prevención de infecciones. En cuidados indirectos se incluyó: administración operacional; calidad y seguridad; coordinación; dirección administrativa; gestión transversal del cuidado; manejo de insumos; registros e informes; y capacitación del personal.

Se seleccionó al azar 70\% de los pacientes, estratificados por UHD y por categoría de dependencia y riesgo. Paralelamente se capacitó y supervisó a un grupo de observadores externos, quienes midieron durante una sesión de HD los tiempos utilizados por la enfermera en ejecutar las actividades, haciendo una estimación general y por perfil de dependencia y riesgo, expresado en minutos/horas/paciente.

El tiempo empleado en los cuidados indirectos se estimó a través de dos grupos focales con enfermeras de las UHD, estableciéndose un promedio para cada actividad expresada en minutos/ horas por paciente, independiente del perfil de los pacientes.

\section{Análisis estadístico}

Se utilizó el programa estadístico SPSS 18.0. Se calcularon medidas de tendencia central para los datos socio-demográficos y cuidados indirectos. Para determinar la carga de trabajo se correlacionaron los perfiles de pacientes según dependencia-riesgo con las actividades realizadas por la enfermera, mediante la prueba de $\chi^{2}$.

\section{Resultados}

La muestra estuvo conformada por 151 pacientes, sin embargo, los análisis consideraron 140 ya que 11 de ellos no fueron categorizados por traslado a otras UHD (10) y un fallecimiento (1).

El promedio de edad fue de 58,2 años, el $55 \%$ eran hombres, un tercio había cursado solo educación básica, y las causas más frecuentes de IRCT eran hipertensión arterial $(42,1 \%)$ y diabetes mellitus (37,9\%). Casi la mitad de los pacientes presentaba sobrepeso u obesidad $(44,3 \%), 90 \%$ se dializaba a través de una fistula arteriovenosa (FAV) y $12,8 \%$ presentaba algún tipo de discapacidad física. El 58,5\% de los pacientes requería tratamiento endovenoso en alguna de las sesiones de diálisis (Tabla 1).

En relación al grado de dependencia y riesgo, los resultados muestran que los pacientes se distribuyeron en cinco de las doce categorías ubicándose la mayoría en mediano riesgo- autosuficiencia parcial $(57,85 \%)$, seguida por alto riesgo-autosuficiencia parcial (19,28\%).

$\mathrm{Al}$ analizar por separado la dependencia y el riesgo, se observa que ningún paciente se sitúa en los extremos de la categorización de riesgo (máximo o bajo riesgo). En cuanto a la dependencia $77,13 \%$ era autosuficiente-parcial y $2,14 \%$ presentaba dependencia total. Esta distribución no presentó diferencias significativas entre las UHD (Tabla 2).

La enfermera ocupa en promedio $36,47 \mathrm{~min}$ por paciente en cuidados directos. Los pacientes clasificados en alto riesgo-dependencia parcial necesitaron en promedio $41,18 \mathrm{~min}$, y en alto riesgo-autosuficiencia parcial en promedio $40,33 \mathrm{~min}$, 
Tabla 1. Características de los pacientes

\begin{tabular}{|c|c|}
\hline & $(n, \%)$ n: 140 \\
\hline Edad en años (promedio, DS) & $58,2 \pm 15.9$ \\
\hline Hombres & $77(55 \%)$ \\
\hline \multicolumn{2}{|l|}{ Nivel educacional } \\
\hline Básica & $41(30 \%)$ \\
\hline Media & $36(25,7 \%)$ \\
\hline Superior & $21(15 \%)$ \\
\hline No reporta & $42(30 \%)$ \\
\hline \multicolumn{2}{|l|}{ Causa IRCT } \\
\hline Hipertensión & $59(42,1 \%)$ \\
\hline Diabetes mellitus & $53(37,9 \%)$ \\
\hline Otras & $28(20 \%)$ \\
\hline \multicolumn{2}{|l|}{ Estado nutricional } \\
\hline Bajo peso & $16(11,4 \%)$ \\
\hline Normo peso & $62(44,3 \%)$ \\
\hline Sobrepeso & $47(33,6 \%)$ \\
\hline Obeso & $15(10,7 \%)$ \\
\hline \multicolumn{2}{|l|}{ Acceso vascular } \\
\hline FAV (nativa o protésica) & $126(90 \%)$ \\
\hline Catéter venoso central & $14(10 \%)$ \\
\hline \multicolumn{2}{|l|}{ Terapia medicamentosa } \\
\hline Eritropoyetina y fierro & $50(35,7 \%)$ \\
\hline Fierro endovenoso & $17(12,1 \%)$ \\
\hline Calcio endovenoso & $15(10,7 \%)$ \\
\hline \multicolumn{2}{|l|}{ Discapacidad física } \\
\hline Ceguera & $9(6,4 \%)$ \\
\hline Amputación de al menos un miembro & $5(3,6 \%)$ \\
\hline Otras & $4(2,9 \%)$ \\
\hline
\end{tabular}

Tabla 2. Categorización de los usuarios según dependencia y riesgo

\begin{tabular}{|lcc|}
\hline Categorización & $\begin{array}{c}\text { n } \\
\text { paciente }\end{array}$ & $\%$ \\
\hline Máximo riesgo-dependencia total & 0 & 0 \\
Máximo riesgo-dependencia parcial & 0 & 0 \\
\hline Máximo riesgo-autosuficiencia parcial & 0 & 0 \\
\hline Alto riesgo-dependencia total & 3 & 2,14 \\
Alto riesgo-dependencia parcial & 14 & 10 \\
\hline Alto riesgo-autosuficiencia parcial & 27 & 19,28 \\
\hline Mediano riesgo-dependencia total & 0 & 0 \\
\hline Mediano riesgo-dependencia parcial & 15 & 10,7 \\
\hline Mediano riesgo-autosuficiencia parcial & 81 & 57,85 \\
Bajo riesgo-dependencia total & 0 & 0 \\
\hline Bajo riesgo-dependencia parcial & 0 & 0 \\
\hline Bajo riesgo-autosuficiencia parcial & 0 & 0 \\
\hline Total & 140 & 99,97 \\
\hline
\end{tabular}

es decir, ambos requirieron en forma estadísticamente significativa mayor tiempo de cuidados directos que las otras categorías (Tabla 3).

Los cuidados directos que requirieron mayor tiempo de enfermera fueron: conexión y desconexión al monitor, valoración y planificación del cuidado, administración de soluciones parenterales y medicamentos, control de complicaciones agudas, cumplimiento de precauciones estándar $\mathrm{y}$ adicionales, y apoyo emocional a los pacientes.

En una sesión de diálisis con seis pacientes la enfermera ocupa 141,6 min en cuidados indirectos. Las actividades en las que invierten más

Tabla 3. Tiempo invertido por enfermeras en cuidado directo según categoría de dependencia y riesgo por paciente

\begin{tabular}{|lccccc|}
\hline Categoría dependencia y riesgo & $\begin{array}{c}\mathbf{n} \\
\text { pacientes }\end{array}$ & $\begin{array}{c}\text { Media } \\
\text { (min) }\end{array}$ & $\begin{array}{c}\text { DS } \\
\text { (min) }\end{array}$ & $\begin{array}{c}\text { Mínimo } \\
\text { (min) }\end{array}$ & $\begin{array}{c}\text { Máximo } \\
\text { (min) }\end{array}$ \\
\hline B2: Alto riesgo-dependencia parcial & 6 & 41,18 & 21,07 & 27,64 & 75,26 \\
\hline B3: Alto riesgo- autosuficiencia parcial & 18 & 40,33 & 9,47 & 25,96 & 55,84 \\
\hline C2: Mediano riesgo-dependencia parcial & 2 & 37,95 & 11,56 & 22,90 & 33,00 \\
\hline C3: Mediano riesgo- autosuficiencia parcial & 80 & 26,44 & 0,76 & 18,26 & 26,97 \\
\hline Total & $\mathbf{1 0 6}$ & $\mathbf{3 6 , 4 7}$ & $\mathbf{1 0 , 7 1}$ & $\mathbf{2 3 , 6 9}$ & $\mathbf{4 7 , 7 6}$ \\
\hline
\end{tabular}


Tabla 4. Tiempos de cuidados indirectos por enfermera para seis pacientes

\begin{tabular}{|lcc|}
\hline Actividad & Tiempo/min & DE \\
\hline Administración operacional & 22,30 & 1,2 \\
\hline Calidad y Seguridad & 28,40 & 7,49 \\
\hline Coordinación de la atención & 26,80 & 4,01 \\
\hline Gestión transversal del cuidado & 31,00 & 5,38 \\
\hline Manejo de insumos y consumos & 14,40 & 6,1 \\
\hline Registros e informes & 18,70 & 3,49 \\
\hline Total & $\mathbf{1 4 1 , 6}$ & \\
\hline
\end{tabular}

*141,6 min equivale a 2,36 h y a 23,6 min por paciente.

tiempo fueron: gestión transversal del cuidado, calidad y seguridad y coordinación de la atención (Tabla 4). La gestión transversal del cuidado considera participación en visita médica, programación de exámenes y tratamientos, y entrega de turno. Calidad y seguridad incluye chequear y registrar calidad del agua tratada, programar heparina, evaluar registros clínicos, monitorear parámetros en las máquinas. Coordinación de la atención considera relación con unidades de apoyo clínico, de rescate médico y emergencias de la unidad (cortes de energía y agua), entre otras.

\section{Discusión}

Los pacientes de este estudio son en su mayoría hombres, con una edad cercana a los 60 años, similar a la población en HDC chilena. El 90\% de los pacientes contaba con una FAV, cifra mayor que la realidad nacional $(72 \%)^{1}$.

En cuanto a la causa de IRCT, estas coinciden con las más frecuentes a nivel nacional: diabetes mellitus e hipertensión arterial ${ }^{1,26,27}$.

Los pacientes de este estudio tienen un mayor grado de riesgo que de dependencia, hallazgo que no es posible contrastar con la literatura de UHD, ya que los estudios no diferencian la dependencia del riesgo, o bien solo estudian la dependencia $^{28,29-33}$. Por otra parte, los estudios que miden dependencia no evalúan a las personas durante la sesión de diálisis sino en su ambiente cotidiano $^{29,31,32}$. La carga de trabajo de la enfermera en las UHD se distribuyó en $60 \%$ cuidados directos y $40 \%$ de cuidados indirectos, similar al estudio realizado por Rauhala y Fargestrom (2007) en un hospital general de Finlandia donde la enfermera dedicaba $76 \%$ al cuidado directo y $24 \%$ al cuidado indirecto ${ }^{8}$ y al de Meza-Galván (2009) en un servicio de medicina donde la mayoría de la jornada la ocupaba en el cuidado directo ${ }^{34}$.

Nuestros resultados son similares a un estudio realizado en Chile que determinó la necesidad de cuidados directos en pacientes hospitalizados según dependencia y riesgo, teniendo presente que el ambiente clínico era diferente y que evaluaron resultados en $24 \mathrm{~h}$ de trabajo enfermera ${ }^{24}$.

Los cuidados directos en que las enfermeras ocupan más tiempo son principalmente la conexión y desconexión, y el apoyo emocional del paciente. Los indirectos más frecuentes se relacionan con la calidad y seguridad del procedimiento, similar a lo reportado por Trepichio ${ }^{28}$.

Esta investigación utiliza un modelo mixto para determinar la carga de trabajo de la enfermera en una UHD, el cual considera las necesidades de los pacientes y las actividades que la enfermera desarrolla durante una sesión de HD, lo que coincide con Bruzzone (2010) quien plantea que categorizar a los pacientes permite conocer el perfil ocupacional, identificar las cargas de trabajo, y comparar la dotación de enfermería con los requerimientos de los pacientes según su nivel de riesgo y dependencia ${ }^{35}$.

Los resultados de este estudio entregan información útil para distribuir los pacientes de manera equitativa en las salas de diálisis considerando su grado de dependencia y riesgo, de manera de evitar la sobrecarga y estrés de los profesionales, lo que puede incidir en la ocurrencia de eventos adversos. Basado en estos resultados los investigadores sugieren mantener la razón enfermera paciente en las UHD chilenas en 1:69.

\section{Limitaciones del estudio}

Los resultados de esta investigación no fue posible compararlos en su totalidad con estudios previos en este tema ya que muchos de ellos eran realizados en unidades médico-quirúrgicas o de cuidados intensivos utilizando en su mayoría instrumentos basados en las actividades que desarrollan las enfermeras y que no diferenciaban la dependencia y el riesgo de los pacientes. 
Agradecimientos: Agradecimiento a directivos, enfermeras y personal de las UHD participantes de este estudio.

\section{Referencias}

1. Sociedad Chilena de Nefrología [homepage on the Internet]. The Association; C1987-2016 Chile [citado el 31 de enero de 2017]. Dr. Hugo Poblete Badal. Registro de diálisis, XXXVI Cuenta de Hemodiálisis Crónica en Chile. (Al 31 de agosto de 2016). Disponible en: http:// www.nefro.cl/site/biblio/registro/22.pdf

2. Ministerio de Salud (MINSAL), Subsecretaria de Salud pública. Guía Clínica Prevención de Enfermedad Renal Crónica, 2010. Disponible en http://www.bibliotecaminsal.cl/wp/wp-content/uploads/2016/04/Prevenci\%C3\%B3n-Enfermedad-Renal-Cr\%C3\%B3nica-Terminal.pdf

3. Fondo Nacional de Salud (FONASA). Cuenta pública 2016). [homepage on the Internet]. https://www.fonasa.cl/sites/fonasa/adjuntos/Resumen \%20Cuenta\%20 P\%C3\%BAblica\%202015 [citado el 31 de enero de 2017].

4. Sturesson A, Ziegert K. Prepare the patient for future challenges when facing hemodialysis: Nurses' experiences. Int J Qual Stud Health Well-being 2014; 9: 22952. http://dx.doi.org/10.3402/qhw.v9.22952.eCollection2014. [PMC free article] [PubMed].

5. Nobahar M, Tamadon MR. Barriers to and facilitators of care for hemodialysis patients; a qualitative study. Journal of Renal Injury Prevention 2016; 5 (1): 39-44. doi:10.15171/jrip.2016.09.

6. Barrios S, Catoni MI, Fernández F. ¿Existe relación entre la dotación de personal de enfermería, eventos adversos y mortalidad?. Enferm Nefrol 2012; 15 (3): 163-9.

7. Mushi L, Marschall P, Flessa S. The cost of dialysis in low and middle-income countries: a systematic review. BMC Health Serv Res 2015;15 (1): 506.

8. Alghamdi MG. Nursing workload: A concept analysis. J Nurs Manag 2016; 24 (4): 449-57. Recuperado de http:// dx.doi.org/10.1111/jonm.12354.

9. Ministerio de Salud. Decreto No 2.357 de 1994, Aprueba Reglamento Sobre Centros de Diálisis Publicada Diario Oficial. Chile. MINSAL; 1994 [citado 2 febrero 2017]. Disponible en: http://web.minsal.cl/sites/default/files/ files/DTO-2357_31-DIC-1994_dialisis.pdf

10. Sociedad Chilena de Nefrología [homepage on the Internet]. The Association; C1987-2010 Chile [citado el 31 de junio de 2017]. Dr. Hugo Poblete Badal. Registro de diálisis, XXX Cuenta de Hemodiálisis Crónica en Chile.
(Al 31 de agosto de 2010). Disponible en: http://www. nefro.cl/site/biblio/registro/22.pdf

11. Gardner J, Walton J. Striving to be heard and recognized: Nurse Solutions for improvement in the outpatient hemodialysis work environment. Nephrol Nurs J 2011; 38 (3): 239-53.

12. Prezerakos P, Galanis P, Moisoglou I. The work environment of haemodialysis nurses and its impact on patients' outcomes. Int J Nurs Pract 2015; 21 (2): 132-40.

13. Barrios $S$, Arechabala MC. Relación entre carga laboral y burnout en enfermeras de unidades de diálisis. Enferm Nefrol 2012; 15 (1): 46-55.

14. Arechabala MC, Barrios S, Catoni MI, Palma E, Ibacache Y, Richards J. Validación de instrumento de medición de cuidados según dependencia y riesgo en pacientes en terapia de hemodiálisis crónica. Aquichán 2014; 14 (4): 473-84.

15. Ministerio de Salud y Acción Social. Ley 22. 853. Programa Nacional de Garantía de Calidad y Atención Médica - Resolución 739/97 Normas de Organización y funcionamiento de la practica dialítica. Recuperado de: file://C:/Users/Silvia/Downloads/ley-22853\%20 Nacional\%20de\%20dialisis.pdf.

16. Asociación de Trabajadores de la Sanidad. Convenio Colectivo de Trabajo 122/75. Recuperado de: http://www. atsa.org.ar/Leyes/CC122/Convenio_Colectivo_122/ index.html.

17. Ministério da Saúde. Portaria no 389, de 13 de março de 2014. 2014. Disponible en: http://pesquisa.in.gov.br/ imprensa/jsp/visualiza/index.jsp?data $=14 / 03 / 2014$ \&jornal $=1$ \&pagina $=34 \&$ totalArquivos $=164>$.

18. RESOLUÇÃO Conselho Federal de Enfermagem (COFEN) 543/2017 2017. Disponible en: http://www.cofen. gov.br/resolucao-cofen-5432017_51440.html.

19. Diario Oficial de la Generalitat 47268 de Catalunya Núm. 5398 (2009) DECRETO 92/2009, de 9 de junio, por el que se establecen los requisitos técnico sanitarios para la autorización sanitaria de los centros y unidades de diálisis equipados con monitores de hemodiálisis. Disponible en: http://benasque.aragob. es:443/cgi-bin/CONV/BRSCGI?CMD=VEROB$\mathrm{J} \& \mathrm{MLKOB}=374581794342$.

20. ORDEN 101/2008, de 14 de febrero, del Consejero de Sanidad, por la que se regulan los requisitos técnico-sanitarios de los proveedores de asistencia sanitaria sin internamiento y de los servicios sanitarios integrados en una organización no sanitaria. Disponible en: http:// www.madrid.org/cs/Satellite?c=CM_Seccion_BOCM\&cid $=1142431194708 \&$ \&idBoletin $=1142431194682 \% 2 \mathrm{C} 1$ $142431194690 \% 2 \mathrm{C} 1142431194694 \% 2 \mathrm{C} 114243119470$ 8\&pagename $=$ ComunidadMadrid\%2FEstructura. 
21. Legifrance. Décret $n^{\circ} 2002-1198$ du 23 septembre 2002 relatif aux conditions techniques de fonctionnement des établissements de santé qui exercent l'activité de traitement de l'insuffisance rénale chronique par la pratique de l'épuration extrarénale et modifiant le code de la santé publique (troisième partie : Décrets) Disponible en: https://www.legifrance.gouv.fr/affichTexte.do?cidTexte=JORFTEXT000000780502\&dateTexte $=\&$ categorieLien=id.

22. Elseviers M, De Vos J-Y, Harrington M, Zampieron A, Ormandy P, Kafkia T. Comparison of renal care practice in Europe: Centre and patient characteristics, EDTNA/ERCA Journal 2006; 32 (1): 8-13.

23. García MA. Categorización de los usuarios del hospital clínico de la Universidad Católica de Chile según dependencia y riesgo. Rev Horiz Enferm 1995; 6 (2): 56-64.

24. García MA, Castillo L. Categorización de Usuarios: Una herramienta para evaluar cargas de trabajo de enfermería. Rev Med Chile 2000; 128 (2): 177-83.

25. García MA, Soto P, Arqués V. Aplicación de la categorización de usuarios en el desarrollo de un modelo de gestión del cuidado de un hospital del Sector Público. Rev Horiz Enferm 2007; 18 (2): 145-51.

26. Ministerio de Salud (MINSAL). Guía Clínica Prevención Enfermedad Renal Crónica. Santiago: Minsal, 2010 Disponible en: http://www.bibliotecaminsal.cl/wp/ wp-content/uploads/2016/04/Prevenci\%C3\%B3n-Enfermedad-Renal-Cr\%C3\%B3nica-Terminal.pdf.

27. Zúñiga $C$, Müller H, Flores M. Prevalencia de enfermedad renal crónica en centros urbanos de atención primaria. Rev Med Chile 2011; 139 (9): 1176-84.

28. Trepichio PB, Guirardello EB, Duran ECM, Brito AP. Patient profile and nursing workload at the nephro- logy unit. Rev Gaúcha Enferm. 2013;34(2):133-139. Disponible en: http://dx.doi.org/10.1590/S198314472013000200017.

29. Cook WL, Jassal SV. Functional dependencies among the elderly on hemodialysis. Kidney Int 2008; 73: 128995.

30. Cobo JL, Pelayo R, Sánchez MS, Alonso R, Menezo R, Gándara, et al. Repercusión del grado de dependencia de los pacientes en hemodiálisis sobre la carga de trabajo de enfermería. Rev Soc Esp Enferm Nefrol 2008; 11 (1): 6-11.

31. Farrokhi F, Jassal SV. Routine use of an abbreviated 4-item scale to assess dependence in essential activities of daily living amongst elderly hemodialysis patients: a validation study. Int Urol Nephrol 2013; 45 (1): 259-64. doi: 10.1007/s11255-012-0360-4. Epub 2013 Jan 1.

32. Dijkstra A, Yönt GH, Korhan EA, Muszalik M, Kędziora-Kornatowska K, Suzuki M. The Care Dependency Scale for measuring basic human needs: an international comparison. J Adv Nurs 2012; 68: 2341-8.

33. Meza Galván, M A. Dotación de recursos humanos de enfermería con base en las necesidades de pacientes hospitalizados. Revista de Enfermería Instituto Mexica del Seguro Social 2009; 17: 153-7.

34. Andreu-Periz L, Puig-Llobet M, Cases-Amenós A. Grado de dependencia de las personas sometidas a hemodiálisis en Cataluña y evolución de su mortalidad. Nefrología (Madrid) 2012; 32 (5): 613-21.

35. Bruzzone P, Ochoa G. Categorización usuaria por riesgo y dependencia, ¿utilidad o rutina? Percepción de enfermería. Tesis. Universidad Austral Valdivia. 2010. Disponible en: http://cybertesis.uach.cl/tesis/uach/2010/ fmb914c/doc/fmb914c.pdf 